Wien klin Mag 2022 · 25:1

https://doi.org/10.1007/s00740-022-00434-3

Angenommen: 10. Januar 2022

(C) The Author(s), under exclusive licence to Springer-Verlag GmbH Austria, ein Teil von Springer Nature 2022

Lärm kann krank machen. Das ist mittlerweile durchaus bekannt und wird auch teilweise bereits erforscht. Lärm kann auch töten. Das erscheint auf den ersten Blick vielen, die sich nicht unmittelbar damit beschäftigen, übertrieben, ist aber ebenfalls bereits länger bekannt. (Literarisch geistreich verarbeitet in "The Nine Taylors" von Dorothy L. Sayers [1]). Das Phänomen ist jedenfalls relevant, da viele Lebensbereiche der Erde mit zunehmender Lautstärke konfrontiert sind und zwar eine scheinbare Gewöhnung eingetreten ist, im Hintergrund allerdings die negativen Folgen dennoch eintreten und Ursache und Wirkung oft gar nicht miteinander in Zusammenhang gebracht werden. Dazu kommt, dass die Ohren als Haupteintrittspforte für die Impulse des Hörsinns im Gegensatz zu den Augen für den Sehsinn nicht so einfach „geschlossen“ werden können.

Untersuchungen zufolge empfinden Menschen andauernden Verkehrslärm als besonders störend und Personen, die an stark befahrenen Straßen leben, haben ein höheres Risiko für Stress, Schlafstörungen, Herz-Kreislauferkrankungen und einen früheren Tod. Der Straßenverkehr ist laut einem Bericht der European Environment Agency (EEA) [2] aus dem Jahr 2020 die Hauptquelle für die geräuschbezogene Umweltverschmutzung, gefolgt von Eisenbahnund Flugverkehr sowie Lärm aus der Industrie.

\section{Lärm im sensiblen Bereich}

Hohe Geräuschpegel sind aber beispielsweise auch im Krankenhausbereich in den vergangenen Jahren als Belastungsfaktor sowohl für Patienten als auch für

Verena Kienast

SpringerMedizin, Wien, Österreich

\title{
Es ist zu laut
}

\section{Die Auswirkungen des Lärms sind weitreichend}

Mitarbeiter erkannt worden. Der Aufenthalt auf der Intensivstation, den viele schwerkranke Patienten in einem scheinbar nicht bewussten Zustand erleben, ist begleitet von einer beträchtlichen Menge an Geräuschen von Überwachungs- und Unterstützungsgeräten, von Alltagsgeräuschen wie lauten Gesprächen, sich öffnenden und schließenden Türen, der maschinellen Zimmerreinigung oder dem Hantieren mit Instrumenten und Gegenständen. Unterschätzt wird dabei die im unterbewussten/unbewussten $\mathrm{Zu}$ stand des Patienten weiterhin bestehende Wahrnehmungsfähigkeit und die daraus resultierende Belastung des vegetativen Nervensystems - abgesehen, davon, dass auch die Mitarbeiter den ziemlich hohen Geräuschpegel als Belastung empfinden.

\section{Achtlosigkeit auch unter Wasser}

So wenig Achtsamkeit oft dieser Lärmbelastung in dem sehr sensiblen Bereich der Intensivstationen geschenkt wird, so achtlos geht man bisher auch im großen Maßstab mit der hohen Geräuschentwicklung im Unterwasserbereich in den Ozeanen um - und bringt damit eine wichtige Kette in unserem Ökosystem durcheinander [3]. Der Schiffsverkehr, Ölbohrplattformen und Druckluftkanonen zur Erdölsondierung ebenso wie militärische Sonare zur Ortung von U-Booten führen dazu, dass die Lebewesen im Wasser, die auf die Ortung durch Schallwellen angewiesen sind, ihre Orientierung verlieren, nicht mehr miteinander kommunizieren können, Gefahren nicht erkennen und sowohl bei Beute- als auch bei Partnersuche ins Leere laufen. Bei Fischen wird zusätzlich die Schwarmstruktur durch- einandergebracht, bei Garnelen wurden Wachstumsstörungen und bei Hummern Zellveränderungen festgestellt, wie die Schweizer NGO OceanCare, die seit 2011 als UN-Sonderberaterin für den Meeresschutz tätig ist, feststellt [4]. Es ist wie beim Menschen: Lärm stresst und schwächt damit das Immunsystem und macht insgesamt anfälliger für Krankheiten. Das geht uns alle an - es wird Zeit, leiser und achtsamer zu sein, meint

Ihre

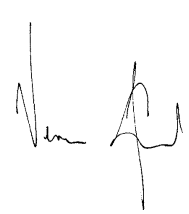

Verena Kienast

\section{Korrespondenzadresse}

Verena Kienast

SpringerMedizin

Wien, Österreich

Verena.kienast@springer.at

\section{Literatur}

1. Sayers DL (1989) The nine taylors. Harcourt Brace Jovanovich, (Reissue edition)

2. www.eea.europa.eu/articles/noise-pollution-isa-major

3. Ö1, 18.12.2021: Strategien gegen UnterwasserLärm. www.oe1.orf.at

4. www.oceancare.org

Hinweis des Verlags. Der Verlag bleibt in Hinblick auf geografische Zuordnungen und Gebietsbezeichnungen in veröffentlichten Karten und Institutsadressen neutral. 